\title{
Optimal Number of Clusters in Dense Wireless Sensor Networks: A Cross-Layer Approach
}

\author{
Li-Chun Wang, Senior Member, IEEE, Chung-Wei Wang, Student Member, IEEE, and \\ Chuan-Ming Liu, Member, IEEE
}

\begin{abstract}
Cluster-based sensor networks have the advantages of reducing energy consumption and link-maintenance cost. One fundamental issue in cluster-based sensor networks is determining the optimal number of clusters. In this paper, we suggest a physical (PHY)/medium access control (MAC)/network (NET) cross-layer analytical approach for determining the optimal number of clusters, with the objective of minimizing energy consumption in a high-density sensor network. Our cross-layer design can incorporate many effects, including lognormal shadowing and a two-slope path loss model in the PHY layer, various MAC scheduling, and multihop routing schemes. Compared with the base-line case with one cluster per observation area (OA), a sensor network with the proposed optimal number of clusters can reduce the energy consumption by more than $80 \%$ in some cases. We also verify by simulations that the analytical optimal cluster number can still effectively function, regardless of the different densities of sensors in various OAs.
\end{abstract}

Index Terms-Cross-layer design, optimal number of clusters, wireless sensor networks.

\section{INTRODUCTION}

C LUSTER-BASED sensor networks have many advantages. For example, with clustering, energy consumption can be improved, because only one representative node per cluster is required to be active, and the other nodes can enter the dormant mode [2]-[4]. Clustering architecture has important applications of high-density sensor networks, because it is much easier to manage a set of cluster representatives from each cluster than to manage whole sensor nodes. Environment measurement [5], target tracking [6], [7], intrusion detection [8], and pursuit-evasion games [9] are typical applications of highdensity sensor networks due to the fault-tolerance requirement.

One of the key challenges in deploying a high-density cluster-based sensor network is determining the optimal number of clusters. For a high-density sensor network, the coverage area can be partitioned into many disjoint spatial coherence

Manuscript received September 13, 2007; revised April 16, 2008. First published July 16, 2008; current version published February 17, 2009. This work was supported in part by the Ministry of Education ATU Plan and in part by the National Science Council, Taiwan, under Contract 96-2221-E-009061. This work was presented in part at the IEEE International Conference on Networks, Sensors, and Control (ICNSC), Taipei, Taiwan, March 2004. The review of this paper was coordinated by Prof. V. Leung

L.-C. Wang and C.-W. Wang are with the Department of Communication Engineering, National Chiao Tung University, Hsinchu 300, Taiwan (e-mail: lichun@cc.nctu.edu.tw; hyper.cm91g@nctu.edu.tw).

C.-M. Liu is with the Department of Computer Science and Information Engineering, National Taipei University of Technology, Taipei 106, Taiwan (e-mail: cmliu@csie.ntut.edu.tw)

Color versions of one or more of the figures in this paper are available online at http://ieeexplore.iee.org.

Digital Object Identifier 10.1109/TVT.2008.928637 regions [10], where the sensed information is highly spatial correlated. In this paper, we call such a spatial coherence region a basic observation area (OA). Intuitively, each basic OA with highly correlated sensed information requires only one cluster representative [11]-[14]. In general, the number of clusters in a basic OA can be determined from different aspects of the protocol layers.

1) From the physical (PHY) layer aspect, using more clusters can save more energy, because the transmission distance between cluster representatives can be shortened.

2) From the medium access control (MAC) layer aspect, having fewer clusters (or, equivalently, more nodes per cluster) decreases the average possibility of being a cluster representative for each sensor node and, thus, reduces energy consumption.

3) From the multihop routing aspect in the network (NET) layer, fewer clusters yield fewer hop counts to the data sink and result in less energy consumption.

Hence, optimizing the number of clusters in a sensor network becomes a cross-layer tradeoff design issue among the required transmission power in the PHY layer, the possibility of being a cluster representative in the MAC layer, and the hop counts in the relay path in the NET layer.

\section{A. Related Work}

The issue of optimizing the number of clusters in a wireless sensor network has been addressed by many researchers from different viewpoints.

1) First, from the viewpoint of propagation distance in the PHY layer, many authors [15]-[20] have discussed how to design the number of clusters in a sensor network. Duarte-Melo and Liu [15] concluded that the number of clusters should be as large as possible, because the distance between the cluster head and its members can be shortened. However, in [16] and [17], it was shown that a larger number of clusters also lead to more onehop transmissions from the heads to the sink. Thus, the optimal number of clusters exists [18], [19]. Furthermore, Depedri et al. [20] discussed the effects of shadowing and path loss exponents on the optimal number of clusters.

2) From the aspect of the MAC layer, Duarte-Melo and Liu [21] suggested that the design of the optimal number of clusters should include the impact of the contention mechanism. However, an explicit MAC protocol for achieving this goal was not presented. 
3) From the routing aspect, Mhatre and Rosenberg [22] assumed that each node sends data to the corresponding cluster heads using multihop routing. They found that more clusters will result in more one-hop transmissions from the heads to the sink although the total routing traffics within each cluster is reduced because of fewer members. Furthermore, Zhou and Abouzeid [23] determined the optimal number of clusters from the viewpoint of minimizing the routing overhead using an informationtheoretical approach.

Current methods for determining the optimal number of clusters are based on each individual protocol layer aspect. To the best of our knowledge, a PHY/MAC/NET cross-layer analytical approach for determining the optimal number of clusters in a dense sensor network has received little notice in the literature.

\section{B. Contributions and Organization of This Paper}

The objective of this paper is to develop a PHY/MAC/NET cross-layer analytical method for calculating the optimal number of clusters in each basic OA for a high-density sensor network. Based on the proposed analytical approach, an optimal number of clusters can be obtained without time-consuming search and labor-intensive field trials. Therefore, the deployment of the energy-efficient cluster-based sensor network becomes easier.

The contributions of this paper can be summarized here.

- First, we propose a cross-layer analytical approach for determining the optimal number of clusters. In the cluster number design problem, it is a concept in the average sense. Hence, we first suggest adopting the criterion of the minimal total average energy (instead of the lifetime in the extreme case) to calculate the optimal number of clusters per OA. The PHY/MAC/NET cross-layer analytical design approach for the optimal number of clusters in high-density sensor networks has not been seen in the literature.

- Second, we show the existence of the optimal cluster number, regardless of the different densities of sensors in various OAs by simulations and analyses. We also take account of other randomness factors in our simulation platform (Fig. 6), including lognormal shadowing and a more realistic two-slope path loss model. The simulation results are shown to match the proposed analytical results quite well. To the best of our knowledge, this interesting finding of the optimal cluster number, regardless of the different densities of sensors in various OAs, has yet to be reported in the literature.

The rest of this paper is organized as follows: In Section II, we introduce the system model and formulate an optimization problem to find the best number of clusters in high-density sensor networks. Section III discusses how to determine the optimal number of clusters from a PHY/MAC cross-layer perspective. In Section IV, we further consider the NET layer aspect. The numerical results are shown in Section V. Finally, we give our concluding remarks in Section VI.

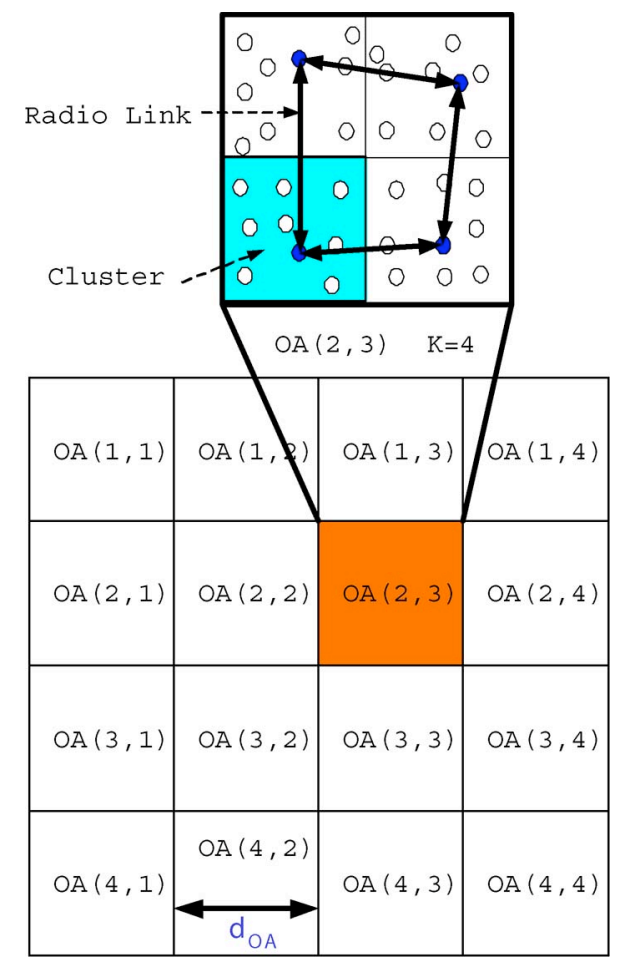

Fig. 1. Sensor network divided into a grid of $M=16$ basic OAs; in each of them, we form $K=4$ clusters. The filled (unfilled) circle represents a cluster representative (dormant) node.

\section{System Model and Problem Formulation}

\section{A. System Model}

Consider a sensor network with $N$ nodes in a grid of $M$ basic OAs, each containing $K$ square-shaped clusters. A basic $O A$ represents a spatial coherence region with highly correlated information. Define $d_{\mathrm{OA}}$ as the minimal distance that results in uncorrelated information [10]. Fig. 1 shows an example of a sensor network with $M=16$ OAs and four clusters per OA (i.e., $K=4$ ). In any instant, only one sensor node per cluster is active to sense the surrounding information, whereas the other nodes enter the dormant mode. Furthermore, we suppose that the sensor nodes in each cluster can be synchronized by a certain clock synchronization mechanism [24].

To ease analysis, the grid model was used for sensor networks [25]-[29]. In general, the shape of the clusters can be arbitrary (or close to circle) because of the randomness inherent in radio propagation. However, the grid model is still a good candidate for obtaining insights on system design [30]. ${ }^{1}$

Now, we briefly introduce the operations of the cluster-based sensor network considered in this paper. First, the clusters are formed according to some cluster formation mechanisms [31]. One cluster head is selected in each cluster to schedule the subsequent cluster representatives from its cluster members. When receiving the schedule broadcast from the cluster head, all sensor nodes wake up to be the cluster representatives

\footnotetext{
${ }^{1}$ To design cluster-based sensor networks, we need some geometric shapes to tessellate the entire coverage area, such as triangle, square, and hexagon. Hexagon-shaped coverage is widely used for a base station in mobile cellular systems. Our approach can also be modified to hexagon-shaped clusters, which is not included in this paper due to the limitation on page length.
} 
in turn. The cluster representatives periodically report sensed information to the sink based on a multihop delivery. Note that the clusters are periodically reformed, and the reformation period of the clusters is called a round in this paper.

\section{B. Problem Formulation}

In most of the literature, the network lifetime is defined as the time that elapsed from the start of the sensor network to the death of the first node [32]. In this case, the researches cared for the maximal energy consumption over all sensor nodes. Hence, the objective function of finding the optimal number of clusters can be expressed as follows:

$$
K_{\mathrm{opt}}=\underset{1 \leq K \leq \frac{N}{M}}{\arg \min } \max _{1 \leq i \leq N} E_{i}(K)
$$

where $E_{i}(K)$ is the energy consumption of sensor $i$ when the number of clusters in each basic OA is $K$.

For a dense sensor network, we believe that the lifetime in the extreme case may not be the only performance measure to be considered. It is very likely that the remaining sensor nodes in a dense sensor network can still form a network, even if one sensor node does not function. Hence, our goal is to optimize the average energy consumption, instead of the lifetime in the extreme case (i.e., the duration for the first sensor running out its energy). In this paper, we formulate the Energy Minimizing Problem for sensor networks as follows: Given a set of system parameters, including the minimal uncorrelated distances $d_{\mathrm{OA}}$, the number of sensor nodes $N$, and the number of basic OAs $M$, find the optimal number of clusters (denoted by $K_{\mathrm{opt}}$ ) in each basic $O A$ to minimize the average energy consumption $[$ denoted by $\bar{E}(K)]$. Formally

$$
K_{\mathrm{opt}}=\underset{1 \leq K \leq \frac{N}{M}}{\arg \min } \bar{E}(K)
$$

subject to

$$
\begin{array}{ll}
0 \leq P_{t} \leq P_{t_{\max }} & \text { (PHY layer) } \\
0 \leq \bar{r} \leq r_{\max } & \text { (PHY/MAC layer) } \\
1 \leq \bar{h} \leq h_{\max } & \text { (NET layer) }
\end{array}
$$

where $P_{t}, \bar{r}$, and $\bar{h}$ are the transmission power, average retransmission times in the MAC layer, and average hop counts from the cluster representatives to the sink, respectively; and $P_{t_{\max }}$, $r_{\max }$, and $h_{\max }$ are the corresponding maximum values for $P_{t}$, $\bar{r}$, and $\bar{h}$, respectively.

Clearly, the number of clusters $K$ in a basic OA affects the performance of a sensor network from the PHY/MAC/NET cross-layer perspectives. Note that $P_{t}, \bar{r}$, and $\bar{h}$ are all functions of $K$. Specifically, we consider the PHY layer propagation model with different path loss exponents and shadowing components.

\section{PHY/MAC CROSS-LAYER ASPECT}

In this section, we discuss the impacts of the transmission power and the MAC scheduling policy on the number of clusters $K$ from the PHY/MAC cross-layer perspectives.

\section{A. Energy Consumption Model}

To begin with, denote $E_{i \rightarrow j}$ as the energy consumption of transmitting data from sensors $i$ to $j$ during each round, where $i \neq j$. Denote $P_{t}$ and $P_{e}$ as the transmission power and electronics power consumption, respectively. Then, according to [18] and [33], it follows that

$$
E_{i \rightarrow j}=a_{i \rightarrow j}\left[\left(P_{e}+P_{t}\right) \cdot t+P_{e} \cdot t\right]=a_{i \rightarrow j} \cdot\left(2 P_{e}+P_{t}\right) t
$$

where $t$ and $a_{i \rightarrow j}$ are the duration in each transmission and the number of times link $i \rightarrow j$ is established during each round, respectively. Here, the energy consumption for sensing is ignored, because it is much lower than that for transmission [34], [35]. Taking the expectation of $E_{i \rightarrow j}$ over all the links from sensors $i$ to $j$, the average energy consumption of each link during each round can be expressed as follows:

$$
\bar{E}_{i \rightarrow j}=\frac{\sum_{i=1}^{N} \sum_{j=1, j \neq i}^{N} E_{i \rightarrow j}}{N(N-1)}=\left(2 P_{e}+P_{t}\right) t \cdot \bar{a}_{i \rightarrow j}
$$

where $\bar{a}_{i \rightarrow j}=\sum_{i=1}^{N} \sum_{j=1, j \neq i}^{N} a_{i \rightarrow j} /(N(N-1))$ is the average number of times each link is established during each round. Usually, $t$ will be a given system parameter. In Sections III-B and $\mathrm{C}$, we discuss how to obtain $P_{t}$ and $\bar{a}_{i \rightarrow j}$.

\section{B. Impact of Transmission Power}

Consider the two-slope path loss model. Let $n_{1}$ and $n_{2}$ be the path loss exponents and $X_{1}$ and $X_{2}$ be the lognormally shadowing components, respectively. At the distance of $d$, the received power $P_{r}$ can be written as

$$
P_{r}= \begin{cases}\frac{P_{t}}{L_{0_{1}}}\left(\frac{d}{d_{0_{1}}}\right)^{-n_{1}} X_{1}, & d \leq d_{t} \\ \frac{P_{t}}{L_{0_{2}}}\left(\frac{d}{d_{0_{2}}}\right)^{-n_{2}} X_{2}, & d>d_{t}\end{cases}
$$

where $L_{0_{1}}$ and $L_{0_{2}}$ are the path losses at reference distances $d_{0_{1}}$ and $d_{0_{2}}$ minus antenna gain, respectively. In (4), the two different slopes $\left(n_{1}\right.$ and $\left.n_{2}\right)$ are adopted before and after distance threshold $d_{t}$, respectively. In general, we have $n_{1}<n_{2}$. This propagation model had been validated in outdoor sensor networks at $868 \mathrm{MHz}$ [36].

Fig. 2 shows a square-shaped basic OA with four clusters $(K=4)$. To guarantee that any two cluster representatives in adjacent clusters can directly be connected, the one-hop transmission distance of each sensor node (which is denoted by $d$ ) must be larger than or equal to $\sqrt{(5 / K)} d_{\mathrm{OA}}$ [2]. In this paper, we assume that the cluster representatives are connected through the multihop communication method when the propagation distance is longer than $\sqrt{(5 / K)} d_{\mathrm{OA}}$. Hence, given a value of $K$, the value of $d$ is either larger than $d_{t}$ or smaller 


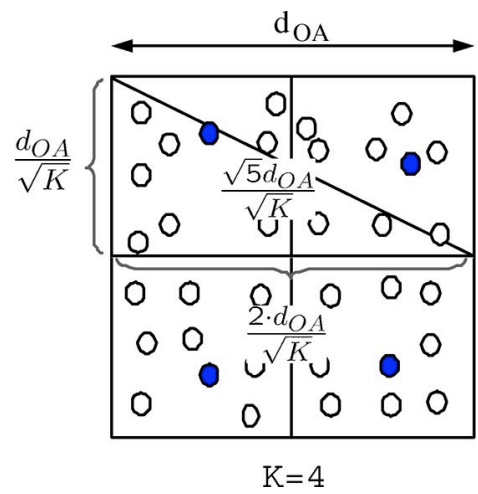

Fig. 2. Square-shaped basic OA with four clusters $(K=4)$, where the maximum one-hop transmission distance between two neighboring cluster representatives is $\sqrt{5} d_{\mathrm{OA}} / \sqrt{K}$ when the side length of a basic $\mathrm{OA}$ is $d_{\mathrm{OA}}$.

than $d_{t}$. Then, $P_{r}$ can uniquely be determined from one of the two particular cases in (4). Without loss of generality, we have

$$
P_{r}=\frac{P_{t}}{L_{0}}\left(\frac{d}{d_{0}}\right)^{-n} X
$$

If $d_{\mathrm{OA}} \leq \sqrt{(K / 5)} d_{t}, L_{0}=L_{0_{1}}, d_{0}=d_{0_{1}}, n=n_{1}$, and $X=X_{1}$. Otherwise, $L_{0}=L_{0_{2}}, d_{0}=d_{0_{2}}, n=n_{2}$, and $X=$ $X_{2}$. In Section V-D, we will evaluate the impact of the twoslope path loss model on the determination of $K$.

From (5), the transmission power of a cluster representative that is needed to maintain the received power level of $P_{r}$ at a distance of $\sqrt{(5 / K)} d_{\mathrm{OA}}$ becomes

$$
P_{t}=P_{r} L_{0}\left(\frac{\sqrt{5} d_{\mathrm{OA}}}{\sqrt{K} d_{0}}\right)^{n}
$$

where the shadowing effect is not considered here (i.e., $X=1$ ) until Section III-E. Hence, from the PHY layer perspective, we know that, with larger values of $K$, the required transmission power of a cluster representative is lower. Note that, because the cluster representatives in adjacent clusters contend for the channel based on the IEEE 802.11 MAC protocol, only one cluster representative can transmit at any time instant. Thus, the intercluster interference does not occur; thus, we do not consider it in (6). The detail of channel contention is discussed in Section IV-C.

\section{Impact of MAC Scheduling}

The MAC layer scheduling policy affects how often cluster representatives $i$ and $j$ establish a link, i.e., $a_{i \rightarrow j}$. A time-driven sensor network usually specifies the number of reports from each cluster during each round, which is denoted by $\bar{R}$. For a sensor network with $M K$ clusters, $M K \bar{R}=\sum_{i=1}^{N} \sum_{j=1, j \neq i}^{N} a_{i \rightarrow j}$. In (3), we have $\bar{a}_{i \rightarrow j}=$ $\sum_{i=1}^{N} \sum_{j=1, j \neq i}^{N} a_{i \rightarrow j} /(N(N-1))$. Thus, it follows that

$$
\bar{a}_{i \rightarrow j}=\frac{M K \bar{R}}{N(N-1)} .
$$

From (7), once $\bar{R}$ is specified, $\bar{a}_{i \rightarrow j}$ in (3) can be obtained.

\section{Optimal Number of Clusters $K_{l}$}

To determine the optimal number of clusters in a basic OA from both the MAC scheduling and PHY transmit power perspectives, we can substitute (6) and (7) into (3). Let $\eta$ be the required receive power. Then, the average energy consumption of each link during each round can be expressed as

$$
\bar{E}_{i \rightarrow j}(K)=\frac{M K}{N(N-1)} \bar{R}\left[2 P_{e}+\eta L_{0}\left(\frac{\sqrt{5} d_{\mathrm{OA}}}{\sqrt{K} d_{0}}\right)^{n}\right] t .
$$

From (8), one can find that $K$ affects the energy consumption in two ways. On the one hand, when $K$ decreases, the number of sensor nodes in a cluster increases. Because a smaller value of $K$ results in more members in a cluster and, thus, lower probability to be a cluster representative, energy consumption can be reduced. On the other hand, a smaller $K$ can also increase the energy consumption of a sensor node due to the longer transmission distance in a larger coverage area. Because $\left(\partial^{2} \bar{E}_{i \rightarrow j}(K) / \partial^{2} K\right)>0,(8)$ is a convex function. Then, letting $\left(\partial \bar{E}_{i \rightarrow j}(K) / \partial K\right)=0$, we can obtain the optimal number of clusters $K_{l}$ to minimize $\bar{E}_{i \rightarrow j}$ from both the PHY and MAC layer perspectives. Specifically, we can express $K_{l}$ as

$$
K_{l}= \begin{cases}\left(\frac{\left(\frac{n}{2}-1\right) \beta}{2 \alpha}\right)^{\frac{2}{n}}, & n>2 \\ 1, & n=2\end{cases}
$$

where $\alpha=P_{e}$, and $\beta=\eta L_{0}\left(\sqrt{5} d_{\mathrm{OA}} / d_{0}\right)^{n}$.

\section{E. Shadowing Effect}

Now, we consider the shadowing effect in (5). Recall that shadowing component $X$ is modeled as a lognormal random variable with zero mean and standard deviation $\sigma$. Thus, the probability density function of the received signal power $P_{r}$ can be expressed as [37]

$$
\begin{aligned}
& f_{P_{r}}(y)=\frac{1}{\sqrt{2 \pi} \sigma\left(\frac{\ln 10}{10}\right) y} \\
& \cdot \exp \left\{-\frac{\left[10 \log _{10} y-10 \log _{10} \frac{P_{t}}{L_{0}}\left(\frac{\sqrt{5} d_{\mathrm{OA}}}{\sqrt{K} d_{0}}\right)^{-n}\right]^{2}}{2 \sigma^{2}}\right\} .
\end{aligned}
$$

Note that the logarithm of the lognormal random variable yields a normal random variable. Define

$$
\begin{aligned}
P_{r(\mathrm{dBm})} & =30+10 \log _{10} P_{r} \\
\eta_{(\mathrm{dBm})} & =30+10 \log _{10} \eta \\
\xi_{(\mathrm{dBm})} & =30+10 \log _{10} \frac{P_{t}}{L_{0}}\left(\frac{d}{d_{0}}\right)^{-n}
\end{aligned}
$$

where "dBm" represents the decibel value normalized to $1 \mathrm{~mW}$, $\eta_{(\mathrm{dBm})}$ is the required received power level, and $\xi_{(\mathrm{dBm})}$ is the 
mean received power without shadowing effect. Accordingly, the outage probability of $P_{r}$ can be calculated as follows:

$$
\begin{aligned}
\operatorname{Pr}\left\{\mathrm{P}_{\mathrm{r}}<\eta\right\} & =\operatorname{Pr}\left\{\operatorname{P}_{\mathrm{r}(\mathrm{dBm})}<\eta_{(\mathrm{dBm})}\right\} \\
& =\int_{-\infty}^{\eta_{(\mathrm{dBm})}} \frac{1}{\sigma \sqrt{2 \pi}} \exp \left[-\left(z-\xi_{(\mathrm{dBm})}\right)^{2} / 2 \sigma^{2}\right] d z \\
& =Q\left(\frac{\xi_{(\mathrm{dBm})}-\eta_{(\mathrm{dBm})}}{\sigma}\right)
\end{aligned}
$$

where

$$
Q(x)=\int_{x}^{\infty} \frac{1}{\sqrt{2 \pi}} \exp ^{-\zeta^{2} / 2} d \zeta
$$

For the outage probability requirement $\theta$, i.e., $\operatorname{Pr}\left\{P_{r}<\eta\right\} \leq$ $\theta$, it follows that

$$
Q^{-1}(\theta) \leq \frac{\xi_{(\mathrm{dBm})}-\eta_{(\mathrm{dBm})}}{\sigma}
$$

Thus, substituting (12) and (13) into (16), we can have

$$
P_{t} \geq \eta L_{0}\left(\frac{\sqrt{5} d_{\mathrm{OA}}}{\sqrt{K} d_{0}}\right)^{n} 10^{Q^{-1}(\theta) \sigma / 10}
$$

Let $P_{t}=\eta L_{0}\left(\sqrt{5} d_{\mathrm{OA}} / \sqrt{K} d_{0}\right)^{n} 10^{Q^{-1}(\theta) \sigma / 10}$ in (17), and substitute (7) into (3). By taking the differential against $K$, for outage probability $\theta$, we can express the optimal number of clusters subject to shadowing as

$$
K_{l}= \begin{cases}\left(\frac{\gamma}{2 P_{e}}\right)^{\frac{2}{n}}, & n>2 \\ 1, & n=2\end{cases}
$$

where $\gamma=((n / 2)-1) \eta L_{0}\left(\sqrt{5} d_{\mathrm{OA}} / d_{0}\right)^{n} 10^{Q^{-1}(\theta) \sigma / 10}$.

In the preceding discussion, we consider the outdoor propagation model validated in [36]. In various environments [38]-[42], $K_{l}$ can also be evaluated by similar approaches.

\section{PHY/MAC/NET LAYER ASPECT}

Based on the PHY/MAC energy consumption model in the previous section, we now further include the effects of channel contention in the MAC layer and routing schemes in the NET layer.

\section{A. Impact of Multihop}

The multihop delivery relies on a number of middle cluster representatives to forward data to the destination. If a sensor node is far away from the sink, the multihop delivery becomes inevitable. The multihop routing scheme consumes less energy in each link than the one-hop delivery [43], but the total energy may also increase due to more relay links.

Assume that sensor $i$ has an $h_{i}$-hop routing path to the sink: $i \rightarrow \gamma_{1} \rightarrow \gamma_{2} \rightarrow \cdots \rightarrow \gamma_{h_{i}-1} \rightarrow \operatorname{sink}$. In this routing path, the energy consumption of link $i \rightarrow j$ in each transmission is
$E_{i \rightarrow j} / a_{i \rightarrow j}$, because $a_{i \rightarrow j}$ is the number of times link $i \rightarrow j$ is established, and $E_{i \rightarrow j}$ is the sum of the energy consumption of link $i \rightarrow j$ during each round. Denote $E(i, \sin k)$ as the sum of the energy consumption from all the sensor nodes in this routing path. Then, we have

$$
\begin{aligned}
E(i, \sin k)=a_{i \rightarrow \gamma_{1}} & \cdot\left\{\frac{E_{i \rightarrow \gamma_{1}}}{a_{i \rightarrow \gamma_{1}}}+\frac{E_{\gamma_{1} \rightarrow \gamma_{2}}}{a_{\gamma_{1} \rightarrow \gamma_{2}}}+\cdots\right. \\
+ & \left.\frac{E_{\gamma_{h_{i}-2} \rightarrow \gamma_{h_{i}-1}}}{a_{\gamma_{h_{i}-2} \rightarrow \gamma_{h_{i}-1}}}+\frac{E_{\gamma_{h_{i}-1} \rightarrow \sin k}}{a_{\gamma_{h_{i}-1} \rightarrow \operatorname{sink}}}\right\} .
\end{aligned}
$$

Usually, the energy consumption of receiving a packet at the sink can be ignored. Hence, we have $E_{\gamma_{h_{i}-1} \rightarrow \operatorname{sink}}=$ $a_{\gamma_{h_{i}-1} \rightarrow \operatorname{sink}} \cdot\left(P_{e}+P_{t}\right) t$. Next, substituting (2) into (19), we can obtain

$$
E(i, \operatorname{sink})=a_{i \rightarrow \gamma_{1}} \cdot\left[\left(h_{i}-1\right) P_{e}+h_{i}\left(P_{e}+P_{t}\right)\right] t .
$$

Averaging over $N$ routing paths, the average energy consumption for each route can be expressed as

$$
\begin{aligned}
\bar{E}(i, \sin k) & =\frac{\sum_{i=1}^{N} E(i, \text { sink })}{N} \\
& =\frac{\sum_{i=1}^{N} a_{i \rightarrow \gamma_{1}}}{N} \cdot\left\{P_{e} \cdot \frac{\sum_{i=1}^{N}\left(h_{i}-1\right)}{N}+\left(P_{e}+P_{t}\right) \cdot \frac{\sum_{i=1}^{N} h_{i}}{N}\right\} t .
\end{aligned}
$$

Recall $\bar{a}_{i \rightarrow j}=\sum_{i=1}^{N} \sum_{j=1, j \neq i}^{N} a_{i \rightarrow j} /(N(N-1))$ in (3). If the first relay node in the routing path from node $i$ to the sink is determined, sensor $i$ transmits to $\gamma_{1}$ only, and $a_{i \rightarrow j}=$ 0 for all $j \neq \gamma_{1}$. In this case, $\bar{a}_{i \rightarrow j}$ is simplified to $\bar{a}_{i \rightarrow j}=$ $\sum_{i=1}^{N} a_{i \rightarrow \gamma_{1}} /(N(N-1))$. Thus, it follows that

$$
\bar{E}(i, \operatorname{sink})=(N-1) \bar{a}_{i \rightarrow j} \cdot\left[P_{e} \cdot(\bar{h}-1)+\left(P_{e}+P_{t}\right) \cdot \bar{h}\right] t
$$

where $\bar{h} \equiv \sum_{i=1}^{N} h_{i} / N$ is the average hop counts for each routing path.

From (22), we observe that a smaller value of $K$ yields less energy consumption, because there are fewer hop counts from the network aspect. However, from the PHY layer aspect, a large $K$ can reduce the transmission power due to the shorter distance. Thus, there exist an optimal number of clusters from the PHY layer transmit power, MAC layer scheduling, and NET layer hop count perspectives. In the next section, we discuss how to obtain $\bar{h}$ based on the shortest-path routing strategy.

\section{B. Calculation of Hop Counts $\bar{h}$}

In this section, we show how to calculate the value of $\bar{h}$ in (22). Although the shortest-path routing is adopted here, the analytical approach can still be applied to other routing strategies. In the considered network, it is assumed that the sink is located at the center of the entire sensor network. As shown in Fig. 3, there are 16 basic OAs with 64 clusters. In this figure, the 


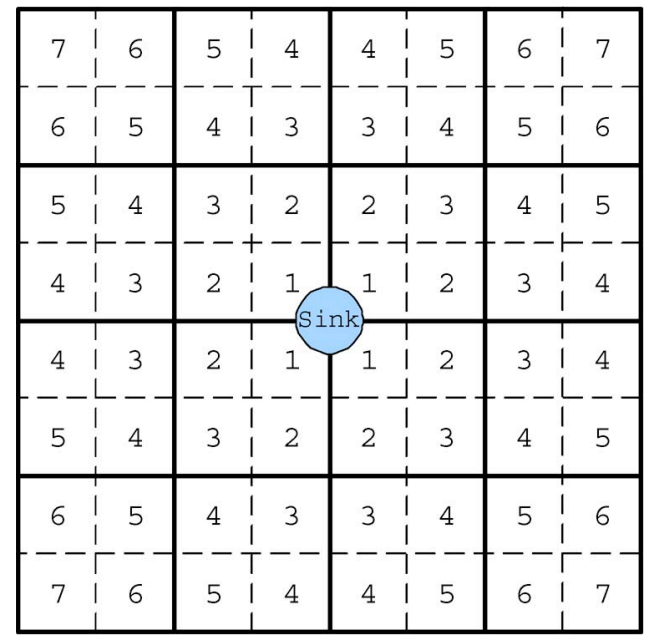

Fig. 3. Sensor network with 64 clusters, where the sink is located at the center of the entire sensor network, and the number in each cluster indicates the required hop counts from the associated cluster to the sink.

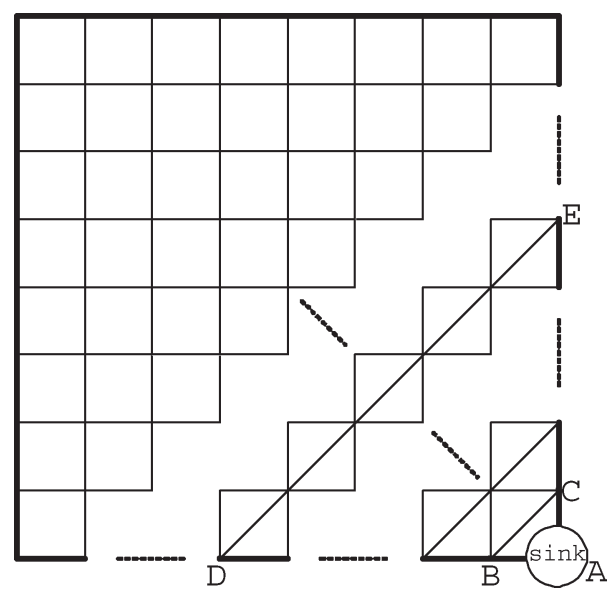

Fig. 4. Top-left portion of the considered sensor network in Fig. 3, where $\left|V^{\prime}(j)\right|$ is proportional to the length of $\overline{D E}$.

number in each cluster indicates the required hop counts to the sink. For example, the hop count of the top-left cluster is seven. Denote $V(h)$ as the set of clusters (or cluster representatives) that can reach the sink within $h$ hops. Thus, $|V(1)|=4$, and $|V(2)|=8$ in Fig. 3. The relation between $|V(h)|$ and $K$ can be calculated by the following lemma.

Lemma 1:

$$
|V(h)|= \begin{cases}4 \cdot h, & 1 \leq h \leq \frac{\sqrt{K M}}{2} \\ 4 \cdot(\sqrt{K M}-h), & \frac{\sqrt{K M}}{2} \leq h \leq \sqrt{K M}-1 .\end{cases}
$$

Proof: From Fig. 3, the pattern of the required hop counts in each cluster is symmetric around the center. Thus, we can consider only the top-left portion, as shown in Fig. 4.

Consider the case of $1 \leq h \leq(\sqrt{K M} / 2)$. Suppose that the set of clusters associated with $\overline{B C}$ is $V^{\prime}(1)$ and that the set of those clusters associated with $\overline{D E}$ is $V^{\prime}(j)$. Thus, we have

$$
\frac{\left|V^{\prime}(j)\right|}{\left|V^{\prime}(1)\right|}=\frac{\overline{D E}}{\overline{B C}}
$$

Clearly, $\left|V^{\prime}(1)\right|=1$. Thus

$$
\left|V^{\prime}(j)\right|=j .
$$

Extending to include all four quadrants, we obtain $|V(h)|=$ $4 h$ when $1 \leq h \leq(\sqrt{K M} / 2)$.

When $(\sqrt{K M} / 2) \leq h \leq \sqrt{K M}-1$, one can also prove that $|V(h)|=4(\sqrt{K M}-h)$.

Theorem 1: The average hop counts $\bar{h}$ from a cluster representative to the sink can be calculated as

$$
\bar{h}= \begin{cases}\frac{\sqrt{K M}}{2}, & \text { when } \sqrt{K M} \text { is an even integer } \\ \frac{\frac{\sqrt{K M}}{2}(K M-1)+1}{K M}, & \text { otherwise. }\end{cases}
$$

Proof: Recall that the total number of cluster representatives is equal to the total number of clusters, i.e., $K M$. Thus, in an area with $K M$ clusters, the largest hop count is equal to $\sqrt{K M}-1$, as observed from Fig. 3. When $\sqrt{K M}$ is an even integer, we have

$$
\begin{aligned}
\sum_{h=1}^{\sqrt{K M}-1}|V(h)| & =\text { the total number of cluster representatives } \\
& =K M
\end{aligned}
$$

In the Appendix, we derive the total hop counts among $K M$ clusters as follows:

$$
\sum_{h=1}^{\sqrt{K M}-1} h \cdot|V(h)|=\frac{(\sqrt{K M})^{3}}{2} .
$$

Combining (27) and (28), we can express the average hop counts for each cluster representative to the sink as

$$
\bar{h}=\frac{\sum_{h=1}^{\sqrt{K M}-1} h \cdot|V(h)|}{\sum_{h=1}^{\sqrt{K M}-1}|V(h)|}=\frac{\sqrt{K M}}{2} .
$$

Applying the same technique to the case in which $\sqrt{K M}$ is an odd integer, one can also prove that

$$
\bar{h}=\frac{\frac{\sqrt{K M}}{2}(K M-1)+1}{K M} \text {. }
$$

\section{Impact of Channel Contention in the MAC Layer}

In this section, we extend (22) to incorporate the effect of channel contention in the MAC layer. Denote $\bar{r}$ as the average retries for transmission in the contention procedures before successfully acquiring a channel. Then, (22) can be extended as

$\bar{E}(i, \operatorname{sink})=(N-1) \bar{a}_{i \rightarrow j}(\bar{r}+1) \cdot\left[P_{e} \cdot(\bar{h}-1)+\left(P_{e}+P_{t}\right) \cdot \bar{h}\right] t$. 
The MAC parameter $\bar{r}$ in (31) is related to the channel quality, the distribution of sensor nodes, the number of clusters, and the handshaking scheme. In this paper, the carrier sense multiple access with collision avoidance (CSMA/CA) MAC protocol is employed under an error-free channel. Based on the CSMA/CA protocol of the IEEE 802.11 standard, we derive the value of $\bar{r}$ as follows:

Let $W$ be the minimum window size. For the maximum backoff stage $m$, the maximum window size is equal to $2^{m} W$. Denote $p_{c}$ as the collision probability when a packet is transmitted on the channel. Assume that each cluster representative always has data to send. According to [44] and [45], in the context of the IEEE 802.11 MAC protocol, the probability that a cluster representative transmits data in a randomly chosen slot time can be expressed as

$$
\tau=\frac{2\left(1-2 p_{c}\right)}{\left(1-2 p_{c}\right)(W+1)+p_{c} W\left(1-\left(2 p_{c}\right)^{m}\right)} .
$$

If there are $q-1$ other cluster representatives within the coverage area of a cluster representative, the collision probability $p_{c}$ for a particular cluster representative can be written as

$$
p_{c}=1-(1-\tau)^{q-1} .
$$

The value of $q$ can be estimated from the number of sensor nodes covered within the transmission range $d$ of a cluster representative. Recall that $d=(\sqrt{5 / K}) d_{\mathrm{OA}}$ in Section III-B. Consider the example shown in Fig. 5, where the cluster representative node $i$ located at $(x, y)$ is inside the center of the square-shaped cluster. There are a total of 25 clusters within the transmission area of cluster representative node $i$. We denote $A_{j}$ as the coverage area of the $j$ th cluster and $B_{j}$ as the area in the $j$ th cluster interfered by the transmission of cluster representative node $i$. Within $A_{j}$, only the cluster representative node in $B_{j}$ will be interfered with cluster representative node $i$. Let $f_{X}(x)$ and $f_{Y}(y)$ be the probability density functions of the sensors' locations in the $x$ - and $y$-axis, respectively. The total average number of contending nodes in 25 clusters can be approximated as follows:

$$
q=\sum_{j=1}^{25} \frac{\iint_{B_{j}} f_{x}(x) f_{Y}(y) d x d y}{\iint_{A_{j}} f_{x}(x) f_{Y}(y) d x d y} .
$$

In the special case when the distribution of the sensor nodes' locations $\left(f_{X}(x)\right.$ and $\left.f_{Y}(y)\right)$ has a self-similar property [46] (e.g., uniform distribution), it follows that

$$
q=25 \cdot \frac{\pi\left(\sqrt{\frac{5}{K}} d_{\mathrm{OA}}\right)^{2}}{\left(\frac{5}{\sqrt{K}} d_{\mathrm{OA}}\right)^{2}}=5 \pi .
$$

Finally, the average retransmission times in the MAC layer can be obtained by substituting (33) into the following equation:

$$
\bar{r}=\sum_{r=0}^{\infty} r \cdot\left(1-p_{c}\right) \cdot\left(p_{c}\right)^{r} .
$$

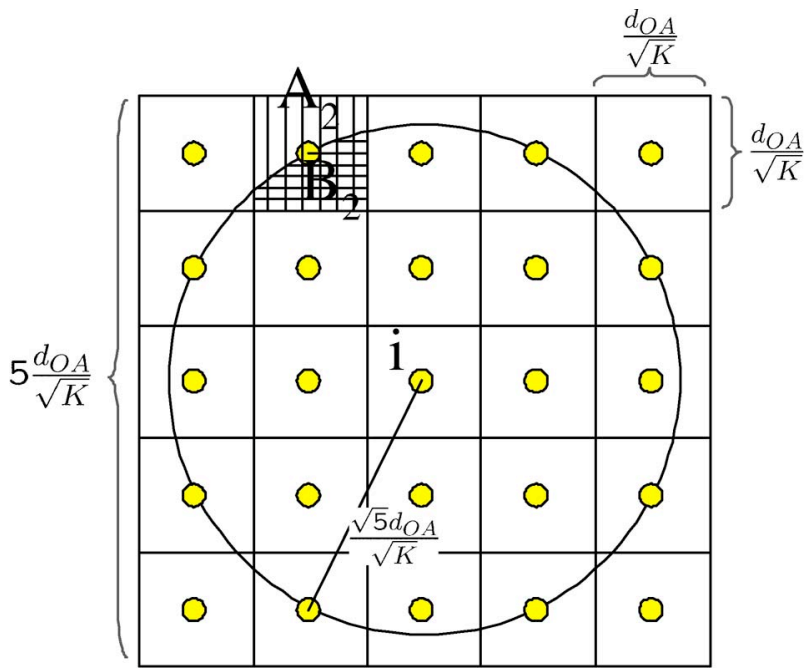

Fig. 5. Transmission area of the cluster representative node located at the center of the cluster. A total of 25 clusters are covered. In this example, $A_{2}$ is the coverage area of the vertical lines, and $B_{2}$ is the coverage area of the horizontal lines.

Note that $\tau$ and $p_{c}$ can iteratively be solved from (32) and (33).

In a more general case when the locations of the sensors are not self similar, $q$ can be a function of $K$. In this case, $\bar{r}$ is also a function of $K$, as described in the constraint in (1). Moreover, if the nonsaturated traffic is considered, $\tau$ and $p_{c}$ must be reevaluated according to the results in the current literature [47]-[51]. Hence, a new average retransmission times $\bar{r}$ can be derived from (36).

\section{Optimal Number of Clusters $K_{p}$}

Without loss of generality, we consider the case in which $\sqrt{K M}$ is an even integer. Substituting (6), (7), and (29) into (22), we have

$$
\begin{aligned}
\bar{E}(i, \sin k)= & \frac{M K}{N} \bar{R} \cdot(\bar{r}+1)\left[P_{e} \cdot\left(\frac{\sqrt{K M}}{2}-1\right)\right. \\
& \left.+\left(P_{e}+\eta L_{0}\left(\frac{\sqrt{5} d_{\mathrm{OA}}}{\sqrt{K} d_{0}}\right)^{n}\right) \cdot \frac{\sqrt{K M}}{2}\right] t
\end{aligned}
$$

where $\bar{r}$ is defined in (36). Taking a differential with respect to $K$ in (37), we can get

$$
\begin{aligned}
\frac{\partial \bar{E}(i, \sin k)(K)}{\partial K}= & \frac{M}{N}(\bar{r}+1) \bar{R} \cdot\left[\left(\frac{3 \sqrt{K M}}{2}-1\right) P_{e}\right. \\
& \left.+\left(\frac{3-n}{4}\right) \eta L_{0}\left(\frac{\sqrt{5} d_{\mathrm{OA}}}{\sqrt{K} d_{0}}\right)^{n}\right] t
\end{aligned}
$$

Because $\left(\partial^{2} \bar{E}(i, \sin k)(K) / \partial^{2} K\right)>0$, (37) is a convex function. Hence, we can obtain the optimal number of clusters in a basic OA $K_{p}$ by finding the root of $\partial \bar{E}(i, \sin k)(K) / \partial K=$ 0 in (38). 


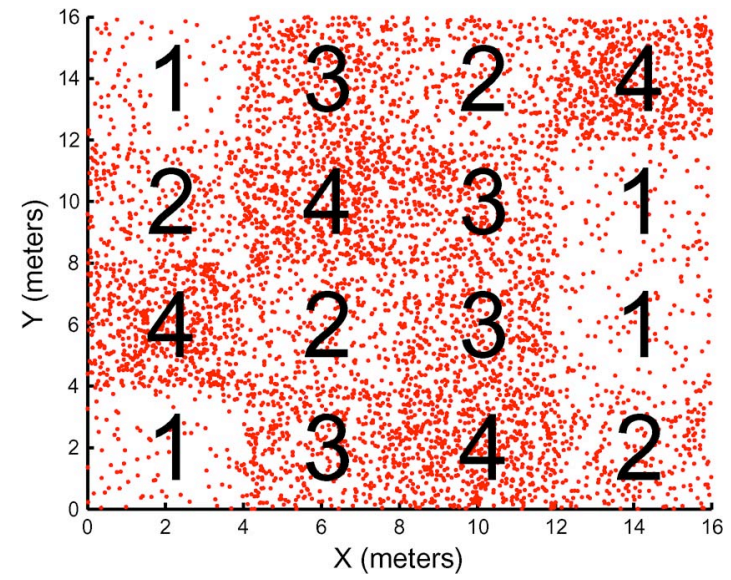

Fig. 6. Simulation scenario.

\section{NumericAl RESUlT}

In this section, we present numerical results to illustrate the relation of the optimal number of clusters and the related parameters in the PHY, MAC, and NET layers. Furthermore, the analytical results are validated through some simulation tests.

\section{A. Simulation Setup}

In the experiment, we consider a $16 \mathrm{~m} \times 16 \mathrm{~m}$ sensor network with 16 basic OAs $(M=16)$. The sensor nodes are uniformly distributed with four kinds of density, as shown in Fig. 6, where the densities of the sensor nodes are 90, 270, 450 , and 630 nodes per OA in regions 1, 2, 3, and 4, respectively. Hence, we have $N=5760$. Furthermore, we first assume that only one set of path loss parameters is needed to obtain the relation between the energy consumption and the number of clusters $K$. We will discuss the impact of the two-slope path loss model in Section V-D. Referring to [52], we adopt the following system parameters: $\bar{R}=5.76 \times 10^{6}, t=10 \mathrm{~ms}$, $P_{e}=5 \mathrm{~mW}, \eta=12.43 \mathrm{pW}, d_{0}=0.2 \mathrm{~m}$, and $L_{0}=52 \mathrm{~dB}$ in (8) and (37).

\section{B. PHY/MAC Layer}

Fig. 7 shows the energy consumption per sensor node against the number of clusters per basic OA according to (8) and the simulation results. As shown, the proposed analytical model can match the simulation results quite well. Furthermore, the optimal number of clusters (which is denoted by the circles in the figure) in a basic OA is $K_{l}=5,30$, and 81 for $n=3,4$, and 5 , respectively. Thus, a sensor network in an environment with a smaller path loss exponent prefers fewer clusters in a basic OA. Second, when $K<K_{l}, E_{\text {link }}$ decreases as $K$ increases, because a smaller $K$ makes the distance between adjacent cluster representatives longer. For $n=4$, the energy consumption per sensor node for the optimal $K_{l}=30$ is reduced to $16.4 \mathrm{~mJ}$, compared with $242.6 \mathrm{~mJ}$ for $K=1$. In this example, the optimal $K_{l}$ can reduce the energy consumption by $93 \%$. Last, when $K>K_{l}$, the energy consumption of the sensor node is proportional to $K$, because the number of times of being the

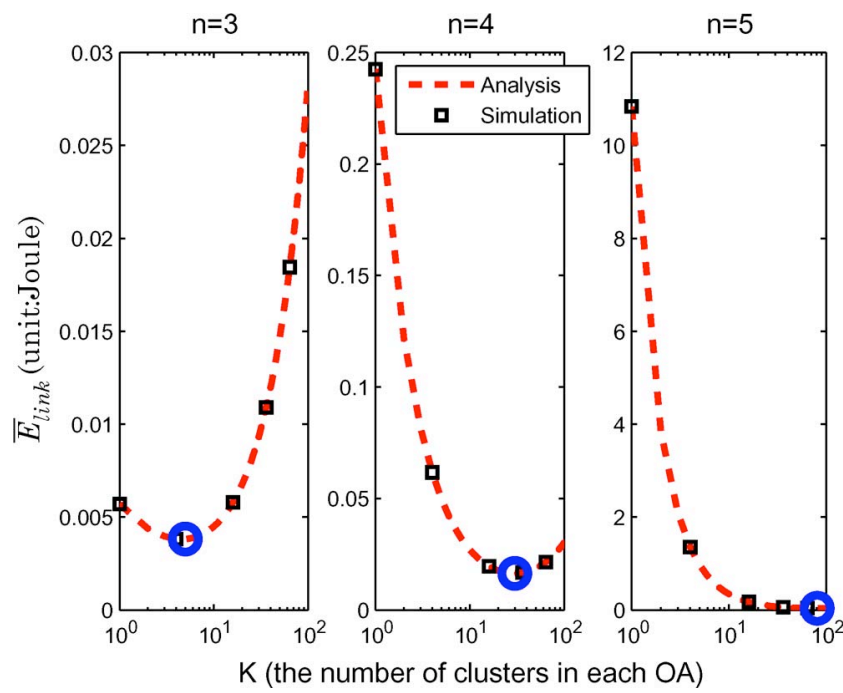

Fig. 7. Energy consumption per sensor node for different numbers of clusters $K$ and path loss exponents $n$, where $\sigma=0 \mathrm{~dB}$. The circle represents the optimal number of clusters.

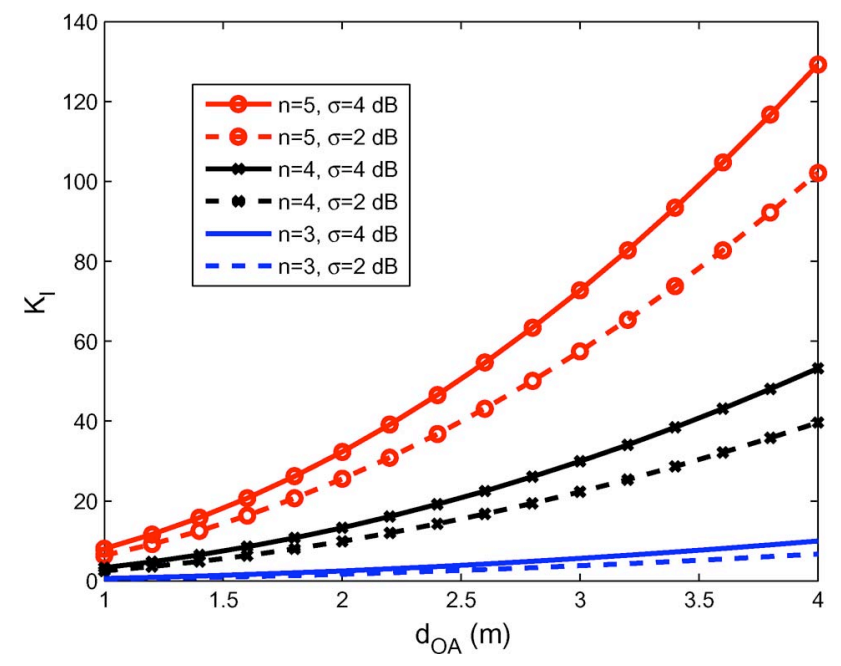

Fig. 8. Impact of $d_{\mathrm{OA}}$ and $\sigma$ on the optimal number of clusters $K_{l}$, where $\theta=0.1$.

cluster representative increases. Compared with $K=60$, the optimal $K_{l}=30$ can decrease the energy consumption from 20.7 to $16.4 \mathrm{~mJ}$, which is equivalent to an energy reduction of $20 \%$.

Fig. 8 shows the impact of the edge length of the basic OA $d_{\mathrm{OA}}$ on the optimal number of clusters $K_{l}$, with $\sigma=2$ and $4 \mathrm{~dB}$. In this example, $\theta=0.1$. We find that, as $\sigma$ increases, $K_{l}$ increases. To overcome more serious shadowing, a shorter transmission distance is preferred, thereby requiring more clusters. Furthermore, the network with fewer basic OAs (or larger $\left.d_{\mathrm{OA}}\right)$ prefers to have more clusters in a basic OA, because more clusters can reduce the transmission distance. Finally, the impact of the shadowing effect and $d_{\mathrm{OA}}$ on $K_{l}$ is more significant for a larger $n$ than a smaller $n$. One can explain this phenomenon from (18). A larger $n$ may amplify the shadowing effect on the optimal number of clusters.

Fig. 9 shows the percentage of energy reduction of the system using the optimal $K_{l}$, compared with the system using 


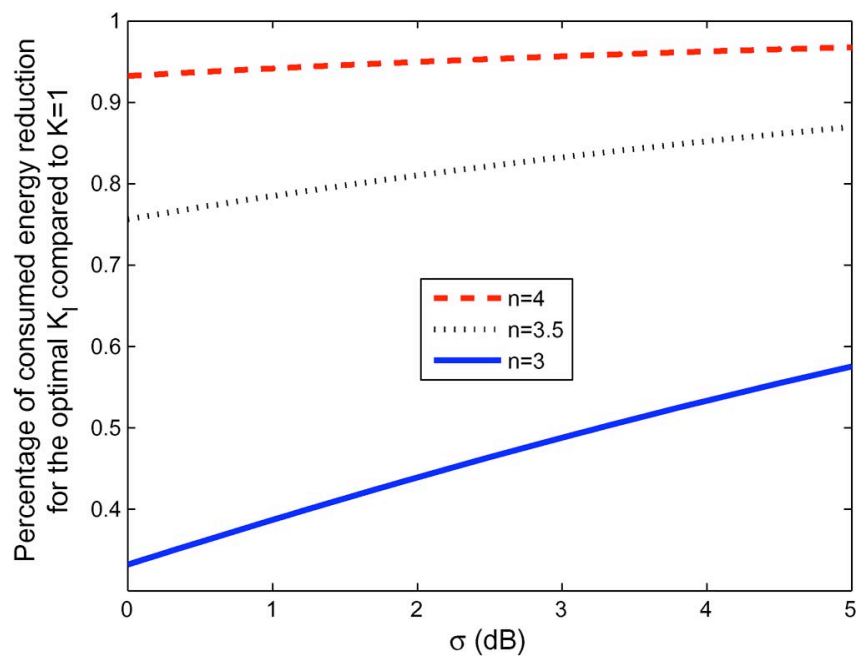

Fig. 9. Energy saving percentage for the optimal $K_{l}$, compared with $K=1$, with the outage probability requirement $\theta=0.1$ for different shadowing standard deviations.

$K=1$ for different shadowing standard deviations. As shown in the figure, the energy saving percentage $\left(1-\left(E_{\text {link }}(K=\right.\right.$ $\left.\left.K_{l}\right) / E_{\text {link }}(K=1)\right)$ increases as path loss exponent $n$ and shadowing standard deviation $\sigma$ increase. For $\sigma=3 \mathrm{~dB}$, the optimal $K_{l}$ can provide energy savings of $48 \%, 83 \%$, and $96 \%$ for path loss exponents $n=3,3.5$, and 4 , respectively.

\section{PHY/MAC/NET Layer}

Fig. 10 shows the relation between the number of clusters $K$ and the total energy consumption of all the sensor nodes in the routing path from a PHY/MAC/NET cross-layer perspective. From this figure, the proposed analytical model can also match the simulation results quite well. Furthermore, when the cluster representatives share the channel without contention (i.e., $\bar{r}=0$ ), the optimal numbers of clusters $K_{p}$ in a basic OA from the PHY/MAC/NET cross-layer perspectives are 1, 17, and 59 for $n=3,4$, and 5, respectively. Comparing Figs. 7 and 10 , we notice that $K_{p}<K_{l}$. This is because $K_{p}$ considers all the PHY/MAC/NET effects. Specifically, fewer clusters yield fewer hop counts to the data sink and result in less energy consumption in the NET layer aspect. Moreover, for a channel with higher collision probability, e.g., $\bar{r}=0.6545$, the total energy consumption increases.

\section{Impact of Two-Slope Path Loss Models}

Now, we further relax the assumption in Figs. 7-10 when only one set of path loss parameters is needed to obtain the relation between the energy consumption and the number of clusters $K$. Now, we consider when two sets of path loss parameters are required to obtain $K$ based on (4). Referring to [36], choose $\left(n_{1}=3, L_{0_{1}}=52 \mathrm{~dB}\right)$ and $\left(n_{2}=4, L_{0_{2}}=44 \mathrm{~dB}\right)$ as the two sets of parameters, respectively, and $d_{t}=0.95 \mathrm{~m}$. Hence, if $K \geq\left(5 d_{\mathrm{OA}} / d_{t}^{2}\right)=22, d \leq d_{t}$, the first set of path loss parameters $\left(n_{1}=3, L_{0_{1}}=52 \mathrm{~dB}\right)$ will be chosen; otherwise, the second set of path loss parameters $\left(n_{2}=4, L_{0_{2}}=44 \mathrm{~dB}\right)$ will be selected. Fig. 11 shows the average energy consumption

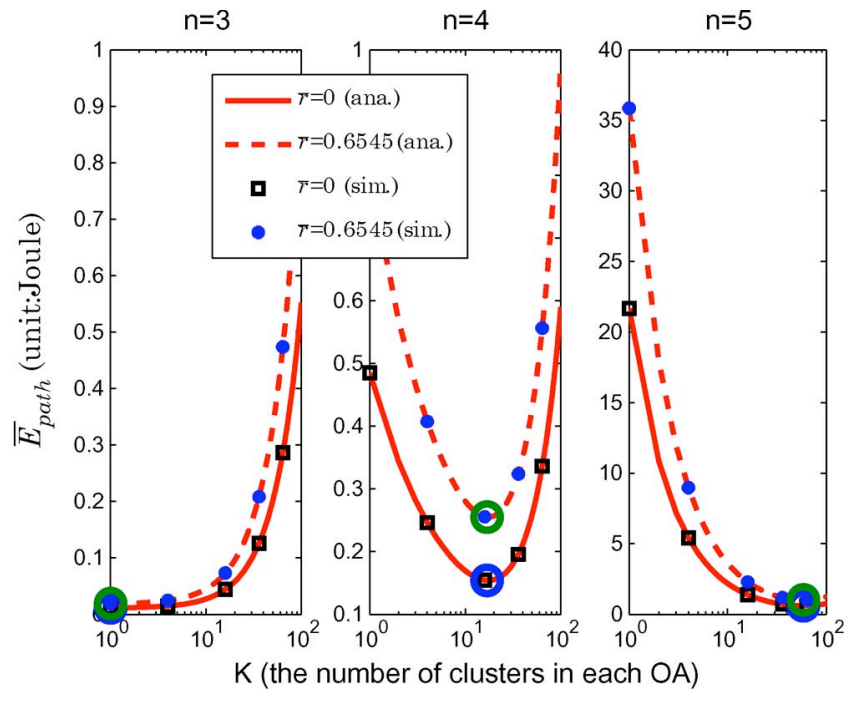

Fig. 10. Energy consumption with $\bar{h}$ hops in different $K$ and $n$ without shadowing. The circle represents the optimal number of clusters.

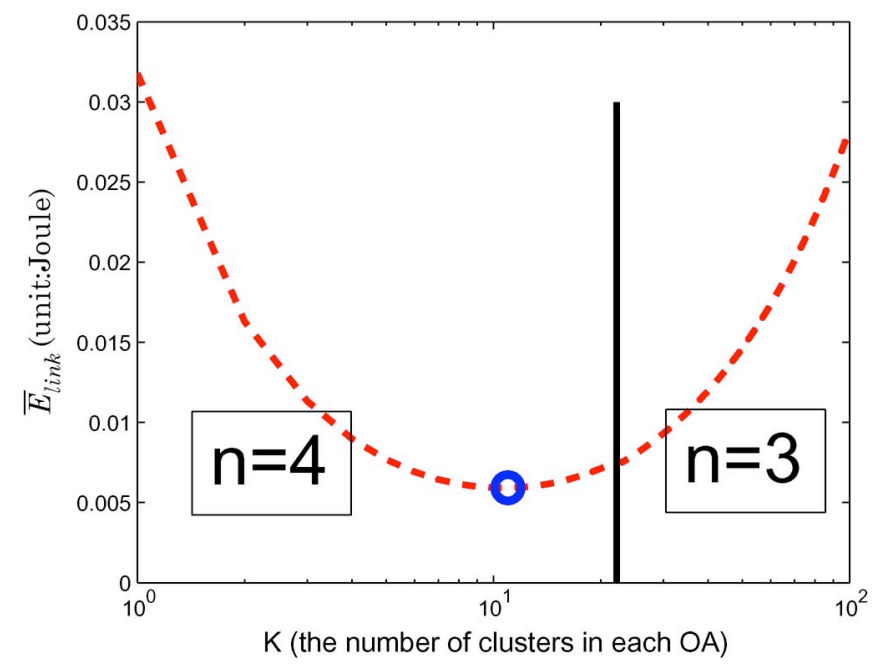

Fig. 11. Energy consumption per sensor node for different numbers of clusters $K$. The circle represents the optimal number of clusters.

versus $K$ for this case. In the figure, the optimal number of clusters in each basic OA is 16 . Note that $K$ will affect the separation distance between two cluster representatives in neighboring clusters. Thus, $K$ will also influence which set of parameters will be selected in the two-slope path loss model. Specifically, a shorter separation distance (or larger $K$ ) is more likely to choose a smaller path loss exponent $n$, which results in better signal strength. That is, from the PHY layer perspective, more clusters in a basic OA are preferred. Compared with $K_{l}=5$ for $n=3$ using the one-slope path loss model in Fig. 7, the two-slope path model leads to a larger value of $K_{l}$.

\section{CONCLUSION}

In this paper, we have presented an analytical approach to determine the optimal number of clusters in a basic OA from the PHY/MAC/NET cross-layer perspectives. Specifically, this cross-layer analytical model integrates the effects of the transmission distance, power, and shadowing in the PHY layer; the 
possibility of being a cluster representative; and the retransmission times in the MAC layer, as well as the number of hops in the NET layer. The closed-form expressions for the optimal number of clusters in a basic OA with various shadowing and path loss conditions are presented. We demonstrate that the suggested analytical optimal number of clusters can significantly improve the energy consumption. With its flexibility, the proposed cross-layer analytical model can facilitate the design of the optimal number of clusters for a sensor network in different radio environments. Furthermore, our simulation results show the existence of the optimal cluster number, regardless of the different densities of sensors in various OAs.

One interesting research topic that can be extended from this paper is the application of a similar methodology to determine the optimal number of clusters based on other MAC and routing strategies with different values of $\bar{r}$ and $\bar{h}$. Furthermore, it is also worthwhile to investigate how to determine the optimal number of clusters when data aggregation and a random traffic model in the upper protocol layers are considered.

\section{APPENDIX}

\section{PROOF OF (28) IN THEOREM 1}

Let $Z=\sqrt{K M} / 2$. Then, we have

$$
\begin{aligned}
\sum_{h=1}^{\sqrt{K M}-1} h \cdot v(h) & =\sum_{h=1}^{Z} h \cdot 4 h+\sum_{h=Z+1}^{2 Z-1}[h \cdot 4(2 Z-h)] \\
& =4 \sum_{h=1}^{Z} h^{2}+8 Z \sum_{h=Z+1}^{2 Z-1} h-4 \sum_{h=Z+1}^{2 Z-1} h^{2} \\
& =8 \sum_{h=1}^{Z} h^{2}+8 Z \sum_{h=Z+1}^{2 Z-1} h-4 \sum_{h=1}^{2 Z-1} h^{2} .
\end{aligned}
$$

Now, we apply the expression for the sum of the series and can derive

$$
\begin{aligned}
\sum_{h=1}^{\sqrt{K M}-1} h \cdot v(h)= & \frac{8}{6} Z(Z+1)(2 Z+1) \\
& +8 Z \frac{[(Z+1)+(2 Z-1)] \cdot(Z-1)}{2} \\
& -\frac{4}{6}(2 Z-1)[(2 Z-1)+1][2(2 Z-1)+1] \\
= & 4 Z^{3} \\
= & \frac{(\sqrt{K M})^{3}}{2} .
\end{aligned}
$$

\section{REFERENCES}

[1] L.-C. Wang and C.-W. Wang, "A cross-layer design of clustering architecture for wireless sensor networks," in Proc. IEEE ICNSC, Mar. 2004, pp. 547-552.

[2] Y. Xu, J. Heidemann, and D. Estrin, "Geography-informed energy conservation for Ad Hoc routing," in Proc. ACM/IEEE Int. Conf. Mobile Comput. Networking, Jul. 2001, pp. 70-84.
[3] A. Cerpa and D. Estrin, "ASCENT: Adaptive self-configuring sensor networks topologies," IEEE Trans. Mobile Comput., vol. 3, no. 3, pp. 272 285, Jul./Aug. 2004.

[4] A. Jindal and K. Psounis, "A clustering method that uses lossy aggregation of data," in Proc. ACM Sensys, Nov. 2004, pp. 269-270.

[5] K. Dasgupta, K. Kalpakis, and P. Namjoshi, "An efficient clustering-based heuristic for data gathering and aggregation in sensor networks," in Proc. WCNC, 2003, vol. 3, pp. 1948-1953.

[6] F. Zhao, J. Liu, J. Liu, L. Guibas, and J. E. Reich, "Collaborative signal and information processing: An information-directed approach," Proc. IEEE, vol. 91, no. 8, pp. 1199-1209, Aug. 2003.

[7] W.-P. Chen, J. C. Hou, and L. Sha, "Dynamic clustering for acoustic target tracking in wireless sensor networks," IEEE Trans. Mobile Comput., vol. 3, no. 3, pp. 258-271, Jul.-Sep. 2004.

[8] Y. Liu, H. Man, and C. Comaniciu, "A game theoretic approach to efficient mixed strategies for intrusion detection," in Proc. IEEE Int. Conf. Commun., 2006, pp. 2201-2206.

[9] B. Sinopoli, C. Sharp, L. Schenato, S. Shaffert, and S. S. Sastry, "Distributed control applications within sensor networks," Proc. IEEE, vol. 91, no. 8, pp. 1235-1246, Aug. 2003.

[10] A. D'Costa, V. Ramachandran, and A. M. Sayeed, "Distributed classification of Gaussian space-time sources in wireless sensor networks," IEEE J. Sel. Areas Commun., vol. 22, no. 6, pp. 1026-1036, Aug. 2004.

[11] M. C. Vuran and I. F. Akyildiz, "Spatial correlation-based collaborative medium access control in wireless sensor networks," IEEE/ACM Trans. Netw., vol. 14, no. 2, pp. 316-329, Apr. 2006.

[12] S. Yoon and C. Shahabi, "The Clustered AGgregation (CAG) technique leveraging spatial and temporal correlations in wireless sensor networks," ACM Trans. Sens. Netw., vol. 3, no. 1, p. 3, Mar. 2007.

[13] H. Gupta, V. Navda, S. R. Das, and V. Chowdhary, "Efficient gathering of correlated data in sensor networks," in Proc. ACM Int. Symp. Mobile Ad Hoc Netw. Comput., 2005, pp. 405-407.

[14] G. Maierbacher and J. Barros, "Source-optimized clustering for distributed source coding," in Proc. GLOBECOM, 2006, pp. 1-5.

[15] E. J. Duarte-Melo and M. Liu, "Energy efficiency in many-to-one communications in wireless networks," in Proc. Midwest Symp. Circuits Syst., Aug. 2002, pp. 615-618.

[16] F. G. Nocetti, J. S. Gonzalez, and I. Stojmenovic, "Connectivity based k-hop clustering in wireless networks," Telecommun. Syst., vol. 22, no. 1-4, pp. 205-220, 2003.

[17] S. Basagni, M. Mastrogiovanni, A. Panconesi, and C. Petrioli, "Localized protocols for ad hoc clustering and backbone formation: A performance comparison," IEEE Trans. Parallel Distrib. Syst., vol. 17, no. 4, pp. 292 306, Apr. 2006

[18] W. B. Heinzelman, A. Chandrakasan, and H. Balakrishnan, "An application-specific protocol architecture for wireless microsensor networks," IEEE Trans. Wireless Commun., vol. 1, no. 4, pp. 660-670, Oct. 2002.

[19] S. Bandyopadhyay and E. J. Coyle, "Minimizing communication costs in hierarchically-clustered networks of wireless sensors," Comput. Netw., vol. 44, no. 1, pp. 1-16, Jan. 2004

[20] A. Depedri, A. Zanella, and R. Verdone, "An energy efficient protocol for wireless ad hoc sensor networks," in Proc. IEEE AINS, Jun./Jul. 2003, pp. 1-6.

[21] E. J. Duarte-Melo and M. Liu, "Analysis of energy consumption and lifetime of heterogeneous wireless sensor networks," in Proc. GLOBECOM, Nov. 2002, pp. 21-25.

[22] V. Mhatre and C. Rosenberg, "Design guidelines for wireless sensor networks: Communication, clustering and aggregation," Ad Hoc Netw. vol. 2, no. 1, pp. 45-63, Jan. 2004.

[23] N. Zhou and A. Abouzeid, "Information-theoretic bounds for mobile adhoc networks routing protocols," in Proc. Int. Conf. Inf. Netw., Oct. 2003, vol. 2662, pp. 651-661.

[24] F. Sivrikaya and B. Yener, "Time synchronization in sensor networks: A survey," IEEE Netw., vol. 18, no. 4, pp. 45-50, Jul./Aug. 2004.

[25] D. Ganesan, D. Estrin, and J. Heidemann, "DIMENSIONS: Why do we need a new data handling architecture for sensor networks?" ACM SIGCOMM Comput. Commun. Rev., vol. 33, no. 1, pp. 143-148, Jan. 2003.

[26] J. N. Al-Karaki, A. E. Kamal, and R. Ul-Mustafa, "On the optimal clustering in mobile ad hoc networks," in Proc. Consum. Commun. Netw. Conf., Jan. 2004, pp. 71-76.

[27] S. Pattem and B. Krishnamachari, "The impact of spatial correlation on routing with compression in wireless sensor networks," ACM Trans. Sens. Netw., vol. V, no. N, pp. 1-31, Sep. 2006.

[28] M. Enachescu, A. Goel, R. Govindan, and R. Motwani, "Scale-free aggregation in sensor networks," Theor. Comput. Sci., vol. 344, no. 1, pp. 1529, Nov. 2005 
[29] A. Scaglione and S. Servetto, "On the interdependence of routing and data compression in multi-hop sensor networks," Wirel. Netw., vol. 11, no. 1/2, pp. 149-160, Jan. 2005.

[30] Principles of Wireless Networks: A Unified Approach, K. Pahlavan and P. Krishnamurthy, Eds. Englewood Cliffs: Prentice-Hall, 2002.

[31] L.-C. Wang, C.-W. Wang, and C.-M. Liu, "Adaptive contention windowbased cluster head election mechanism for wireless sensor networks," in Proc. IEEE VTC, Sep. 2005, vol. 3, pp. 1819-1823.

[32] W. R. Heinzelman, A. Chandrakasan, and H. Balakrishnan, "Energyefficient communication protocol for wireless microsensor networks," in Proc. IEEE Annu. Hawaii Int. Conf. Syst. Sci., Jan. 2000, pp. 3005-3014.

[33] E. Shih, S. Cho, F. S. Lee, B. H. Calhoun, and A. Chandrakasan, "Design considerations for energy-efficient radios in wireless microsensor networks," J. VLSI Signal Process., vol. 37, no. 1, pp. 77-94, 2004.

[34] A. Sawides, S. Park, and M. B. Srivastava, "On modeling networks of wireless microsensors," in Proc. ACM SIGMETRICS Int. Conf. Meas. Model. Comput. Syst., 2001, pp. 318-319.

[35] [Online]. Available: http://www.xbow.com/

[36] A. Martinez-Sala, J.-M. Molina-Garcia-Pardo, E. Egea-Lopez, J. ValesAlonso, L. Juan-Llacer, and J. Garcia-Haro, "An accurate radio channel model for wireless sensor networks simulation," J. Commun. Netw., vol. 7 , no. 4, pp. 401-407, Dec. 2005.

[37] Wireless Communications: Principles and Practice, 2nd ed. T. S. Rappaport, Ed. Englewood Cliffs, NJ: Prentice-Hall, 2002.

[38] J. Zhao and R. Govindan, "Understanding packet delivery performance in dense wireless sensor networks," in Proc. Conf. Embedded Netw. Sens. Syst., 2003, pp. 1-13.

[39] S. Willis and C. Kikkert, "Radio propagation model for long-range ad hoc wireless sensor network," in Proc. Int. Conf. Wirel. Netw., Commun. Mobile Comput., Jun. 2005, vol. 1, pp. 826-832.

[40] A. Cerpa, J. L. Wong, L. Kuang, M. Potkonjak, and D. Estrin, "Statistical model of lossy links in wireless sensor networks," in Proc. Inf. Process. Sens. Netw., Apr. 2005, pp. 81-88.

[41] G. Zhou, T. He, S. Krishnamurthy, and J. A. Stankovic, "Impact of radio irregularity on wireless sensor networks," in Proc. Int. Conf. Mobile Syst., 2004, pp. 125-138.

[42] M. Zuniga and B. Krishnamachari, "Analyzing the transitional region in low power wireless links," in Proc. IEEE Sens. Ad Hoc Commun. Netw. Conf., Oct. 2004, pp. 517-526.

[43] S. Lindsey and C. Raghavendra, "PEGASIS: Power-efficient gathering in sensor information systems," in Proc. IEEE Aerosp. Conf., Mar. 2002, pp. 9-16.

[44] G. Bianchi, "Performance analysis of the IEEE 802.11 distributed coordination function," IEEE J. Sel. Areas Commun., vol. 18, no. 3, pp. 535-547, Mar. 2000.

[45] Y. C. Tay and K. C. Chua, "A capacity analysis for the IEEE 802.11 MAC protocol," Wirel. Netw., vol. 7, no. 2, pp. 159-171, Mar./Apr. 2001.

[46] Real Analysis: Measure Theory, Integration, and Hilbert Spaces, E. M. Stein and R. Shakarchi, Eds. Princeton, NJ: Princeton Univ. Press, 2005.

[47] K. Duffy, D. Malone, and D. J. Leith, "Modeling the 802.11 distributed coordination function in non-saturated conditions," IEEE Commun. Lett., vol. 9, no. 8, pp. 715-717, Aug. 2005

[48] P. E. Engelstad and O. N. Osterbo, "Non-saturation and saturation analysis of IEEE 802.11e EDCA with starvation prediction," in Proc. Int. Workshop Model. Anal. Simul. Wirel. Mobile Syst., 2005, vol. 9, pp. 224-233.

[49] O. Abu-Sharkh and A. H. Tewfik, "Performance analysis of multi-rate 802.11 WLANs under finite load and saturation conditions," in Proc. IEEE VTC, 2005, pp. 2652-2657.

[50] M. Ergen and P. V. Baris Dundar, "Throughput analysis of an extended service set in IEEE 802.11," in Proc. GLOBECOM, Nov. 2004, vol. 2, pp. 1040-1045.
[51] P. C. Ng and S. C. Liew, "Offered load control in IEEE 802.11 multi-hop ad-hoc networks," in Proc. MASS, Oct. 2004, pp. 80-89.

[52] C. Buratti, A. Giorgetti, and R. Verdone, "Simulation of an energy efficient carrier sensing multiple access protocol for clustered wireless sensor networks," in Proc. IWWAN, May/Jun. 2004, pp. 325-329.

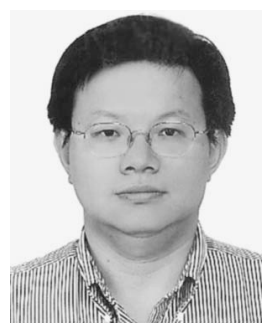

Li-Chun Wang (S'92-M'96-SM'06) received the B.S. degree from National Chiao-Tung University, Hsinchu, Taiwan, in 1986, the M.S. degree from National Taiwan University, Taipei, Taiwan, in 1988, and the M.Sc. and Ph.D. degrees from Georgia Institute of Technology, Atlanta, in 1995 and 1996, respectively, all in electrical engineering.

In 1995, he was with Northern Telecom, Richardson, TX. From 1996 to 2000, he was with the Wireless Communications Research Department, AT\&T Laboratories, where he was a Senior Technical Staff Member. In August 2000, he joined the Department of Communication Engineering, National Chiao-Tung University, as an Associate Professor. In August 2005, he was promoted to Full Professor. He is the holder of three U.S. patents, with three more pending. His current research interests include cellular architectures, radio network resource management, and cross-layer optimization for cooperative and cognitive wireless networks.

Dr. Wang was a corecipient of the Jack Neubauer Best Paper Award from the IEEE Vehicular Technology Society in 1997.

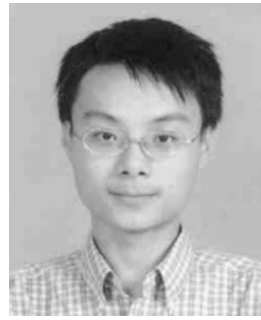

Chung-Wei Wang (S'07) received the B.S. degree in electrical engineering from Tamkang University, Taipei, Taiwan, in 2003. He is currently working toward the Ph.D. degree with the Department of Communication Engineering, National Chiao Tung University, Hsinchu, Taiwan.

His current research interests include cross-layer optimization and MAC protocol design in wireless sensor networks, ad hoc networks, and cognitive radio networks.

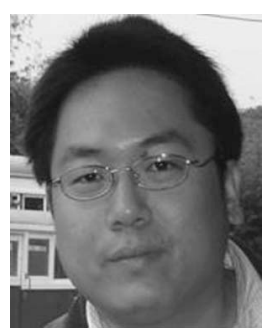

Chuan-Ming Liu (M'03) received the B.S. and M.S. degrees in applied mathematics from National Chung-Hsing University, Taichung, Taiwan, in 1992 and 1994, respectively, and the Ph.D. degree in computer sciences from Purdue University, West Lafayette, IN, in 2002.

He is currently an Associate Professor with the Department of Computer Science and Information Engineering, National Taipei University of Technology, Taipei, Taiwan. His research interests include parallel and distributed computation, data management and data dissemination in wireless environments, ad hoc and sensor networks, and analysis and design of algorithms.

Prof. Liu has been a Member of the Upsilon Pi Epsilon Honor Society in Computer Science since 1998. 